\title{
Use of 3D CT-based navigation in minimally invasive lateral lumbar interbody fusion
}

\author{
Jacob R. Joseph, MD, ${ }^{1}$ Brandon W. Smith, MD, MS, ${ }^{1}$ Rakesh D. Patel, MD, ${ }^{2}$ and Paul Park, MD1 \\ Departments of ${ }^{1}$ Neurosurgery and ${ }^{2}$ Orthopedic Surgery, University of Michigan, Ann Arbor, Michigan
}

OBJECTIVE Lateral lumbar interbody fusion (LLIF) is an increasingly popular technique used to treat degenerative lumbar disease. The technique of using an intraoperative cone-beam CT (iCBCT) and an image-guided navigation system (IGNS) for LLIF cage placement has been previously described. However, other than a small feasibility study, there has been no clinical study evaluating its accuracy or safety. Therefore, the purpose of this study was to evaluate the accuracy and safety of image-guided spinal navigation in LLIF.

METHODS An analysis of a prospectively acquired database was performed. Thirty-one consecutive patients were identified. Accuracy was initially determined by comparison of the planned trajectory of the IGNS with post-cage placement intraoperative fluoroscopy. Accuracy was subsequently confirmed by postprocedural CT and/or radiography. Cage placement was graded based on a previously described system separating the disc space into quarters.

RESULTS The mean patient age was 63.9 years. A total of 66 spinal levels were treated, with a mean of 2.1 levels (range 1-4) treated per patient. Cage placement was noted to be accurate using IGNS in each case, as confirmed with intraoperative fluoroscopy and postoperative imaging. Sixty-four (97\%) cages were placed within Quarters 1 to 2 or 2 to 3 , indicating placement of the cage in the anterior or middle portions of the disc space. There were no instances of misguidance by IGNS. There was 1 significant approach-related complication (psoas muscle abscess) that required intervention, and 8 patients with transient, mild thigh paresthesias or weakness.

CONCLUSIONS LLIF can be safely and accurately performed utilizing iCBCT and IGNS. Accuracy is acceptable for multilevel procedures.

http://thejns.org/doi/abs/10.3171/2016.2.SPINE151295

KEY WORDS image-guided spinal navigation; lateral lumbar interbody fusion; DLIF; XLIF; minimally invasive spine surgery; O-arm Surgical Imaging System

$\mathrm{T}$ HE lateral lumbar interbody fusion (LLIF) technique was initially described by Ozgur et al. in 2006.11 LLIF has alternatively been described as extreme lateral interbody fusion or direct lateral interbody fusion. ${ }^{6,11}$ This minimally invasive retroperitoneal transpsoas approach allows for a more robust discectomy than traditional posterior approaches, while causing minimal blood loss and decreased postoperative pain. ${ }^{5}$ However, the operative approach does place the surgeon directly adjacent to the lumbosacral plexus. To avoid nerve injury, neuromonitoring and fluoroscopy are routinely used, which can result in significant radiation exposure to the surgeon. ${ }^{1,14}$ Image-guided spinal navigation has been well-described for pedicle screw placement and has been shown both to increase accuracy and decrease radiation exposure. ${ }^{9,10,15}$ We have previously shown the feasibility of using intraoperative cone-beam CT (iCBCT) with an image-guided navigation system (IGNS) for cage placement in LLIF. ${ }^{12}$ The objective of this study was to evaluate the accuracy of cage placement and complications in a consecutive series of patients in whom this technique was used.

\section{Methods}

Retrospective analysis of a prospectively acquired database was performed to identify patients who underwent LLIF using the O-arm Surgical Imaging System (Medtronic, Inc.) for 3D image acquisition in conjunction with the

ABBREVIATIONS ASIS = anterior superior iliac spine; iCBCT = intraoperative cone-beam CT; IGNS = image-guided navigation system; LFCN = lateral femoral cutaneous nerve; LLIF = lateral lumbar interbody fusion; PSIS = posterior superior iliac spine.

SUBMITTED October 30, 2015. ACCEPTED February 17, 2016.

INCLUDE WHEN CITING Published online April 22, 2016; DOI: 10.3171/2016.2.SPINE151295. 
StealthStation Surgical Navigation System (Medtronic, Inc.) for spinal navigation. The electronic medical records were reviewed to obtain further data. All patients who underwent LLIF using image-guided spinal navigation were included in the analysis. This study was approved by the University of Michigan Institutional Review Board.

\section{Surgical Technique}

We previously detailed the technique by which the procedure is performed..$^{12}$ Briefly, after the induction of general anesthesia, neuromonitoring leads are placed to monitor somatosensory evoked potentials, and electromyography in addition to motor evoked potentials are used for deformity cases. The patient is positioned on a Jackson flat table in the lateral decubitus position with an axillary roll, in addition to a large flank roll, to open the region between the lower rib cage and iliac crest. Then, the patient is secured at multiple points using tape and prepared and draped in the usual fashion (Fig. 1). Extra care is taken to ensure that the anterior superior iliac spine (ASIS) or posterior superior iliac spine (PSIS) is kept in the sterile field. A stab incision is made over either the ASIS or PSIS, and an iliac pin is impacted into the bone. The IGNS reference arc is attached to the pin. The field is then covered by a second layer of sterile drapes, and the iCBCT unit is positioned to acquire a 3D image of the targeted spinal levels. Up to 4 disc levels can be obtained with a single image acquisition. The 3D image is autoregistered to the IGNS. After registration, the iCBCT unit is removed from the surgical field, and the secondary sterile cover is removed from the patient. Utilizing the navigated initial dilator and trajectory views shown on the IGNS, an incision is demarcated on the skin. After incision, dissection through the subcutaneous tissues is performed and a muscle-splitting approach is used to bluntly traverse the abdominal musculature to enter the retroperitoneal space. The navigated dilator, which also functions as a nerve stimulator, is advanced via image-guided spinal navigation through the psoas muscle into the disc space (Fig. 2). Of note, on multilevel cases, the most superior disc space is targeted first to minimize alteration of the registration on the inferior (distal) segments. Serial dilation is performed once the initial dilator is advanced and secured in the disc space using spinal guidance, followed by placement of an expandable

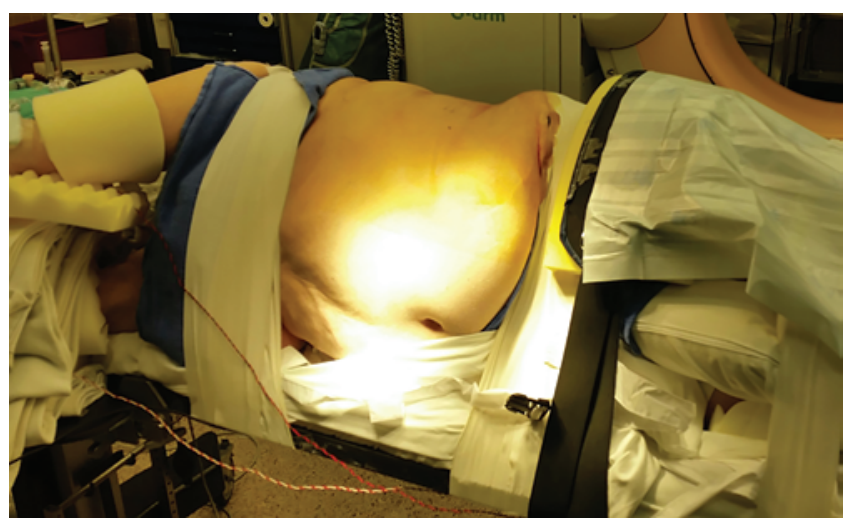

FIG. 1. A patient positioned on a flat Jackson table. Figure is available in color online only.

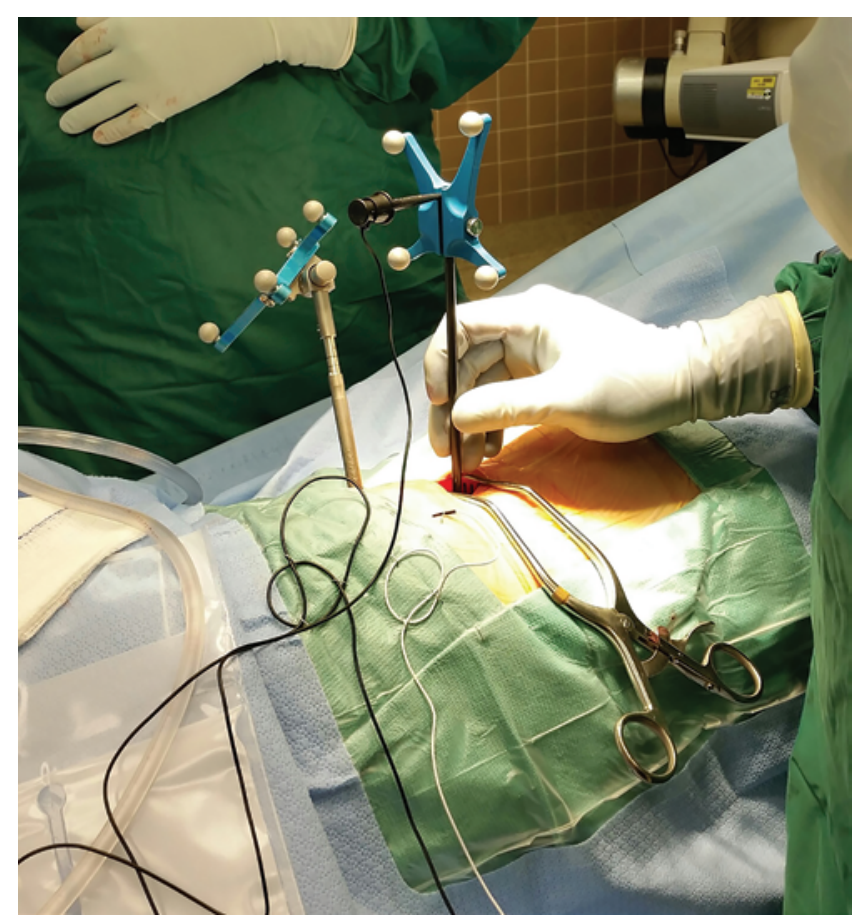

FIG. 2. Navigated dilator with nerve stimulation (clip electrode) used to traverse the psoas muscle and enter the disc space via IGNS instead of fluoroscopic guidance. After "anchoring" the dilator in the disc space, sequential dilation is performed followed by placement of the expandable tubular retractor. Figure is available in color online only.

retractor (Fig. 3). The disc space is directly visualized. A discectomy is performed in the standard manner. Of note, the navigated dilator is used as a pointer with the IGNS, when needed, to confirm localization and trajectory. At this point, the fluoroscope is brought into the field. A complete discectomy is then performed, and the contralateral annulus is released using a Cobb elevator. With the use of the Cobb elevator and disc preparation instruments, fluoroscopy is used as needed since the instruments are not navigated. After discectomy and endplate preparation, a navigated trial is used to size the implant. An appropriately sized cage filled with allograft bone and a biological is then impacted into the disc space via image-guided navigation (Fig. 4). Appropriate cage positioning is confirmed with fluoroscopy.

\section{Radiographic Assessment}

All patients underwent postoperative $\mathrm{CT}$ imaging or postprocedural iCBCT intraoperatively to confirm cage placement. Postoperative radiographs were also obtained in all patients (Fig. 5). Cage placement was graded, as described previously. ${ }^{12}$ In this system, the disc space is separated into quarters, with Quarter 1 representing the anterior 25\% of the disc space and Quarter 4 representing the posterior $25 \%$ of the disc space. Given the width of lateral cages, the location of each cage spanned 2 quarters of the disc space.

\section{Outcome Assessment}

Accuracy was graded intraoperatively by the prima- 


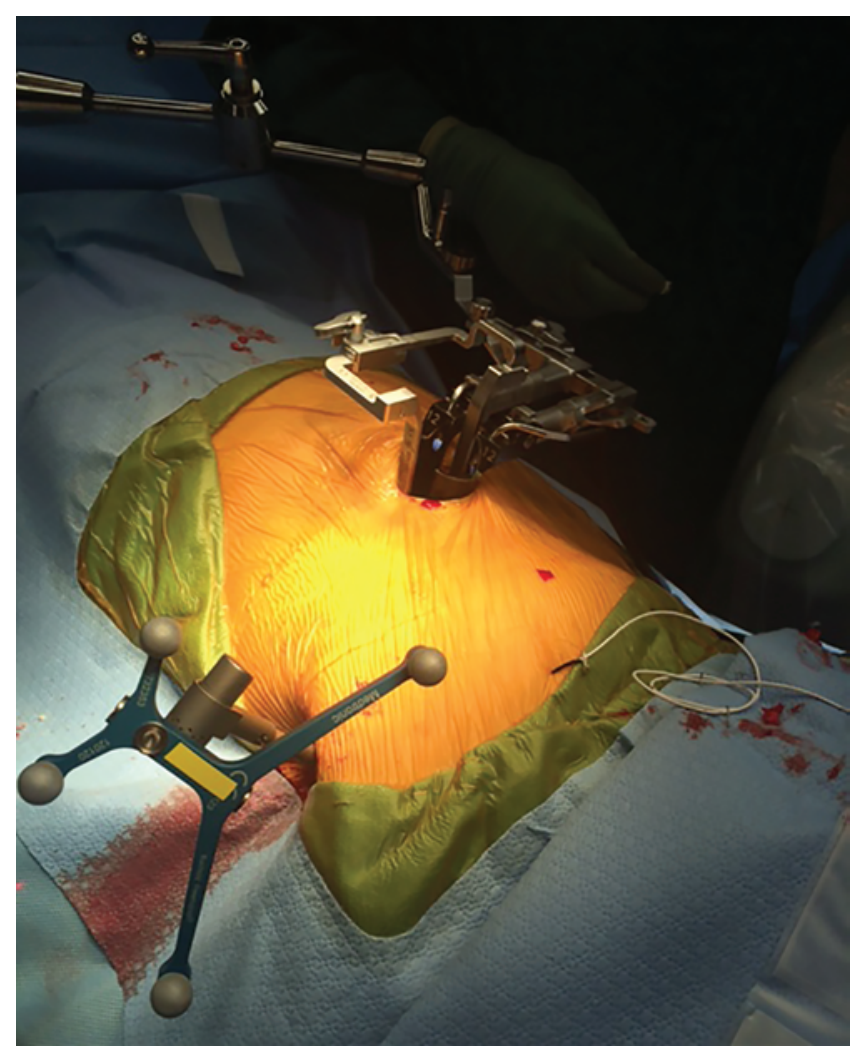

FIG. 3. Final retractor placement using IGNS. Figure is available in color online only. ry surgeon by correlating placement via the IGNS with subsequent intraoperative fluoroscopic images. This was further confirmed postprocedurally with CT or iCBCT as well as radiographs, as described above. There was a minimum of 30 days of clinical follow-up for all patients, during which all medical and surgical complications were recorded. Some patients underwent a staged approach beginning with LLIF followed by further posterior surgery, which in specific cases were performed on a separate operative day. Complications attributed to the second-stage posterior surgery (i.e., wound infection) were not included in this study, as they were not related to the LLIF procedure.

\section{Results}

Table 1 details the patient demographic and procedural data. A total of 31 consecutive patients underwent the procedure. The mean age was 63.9 years (range $24-79$ years). Ten patients were male and 21 patients were female. The average body mass index was $31.0 \mathrm{~kg} / \mathrm{m}^{2}$ (range 24.2-47.1 $\mathrm{kg} / \mathrm{m}^{2}$ ). A total of 66 levels were fused (mean 2.1 levels fused per patient; range 1-4 levels fused per patient). Thirteen patients underwent single-level fusions, while 18 had multilevel fusions (Fig. 5). Preoperative diagnoses included degenerative kyphoscoliosis, idiopathic scoliosis, adjacent segment disease, and pseudoarthrosis. Twenty-six procedures were performed using a left-sided approach, and 5 procedures were performed via a right-sided approach. A single iCBCT image was acquired for each patient, and no reregistration was required. Nine patients had approachrelated complications; 8 of these complications consisted of transient, mild paresthesias or hip flexion weakness.

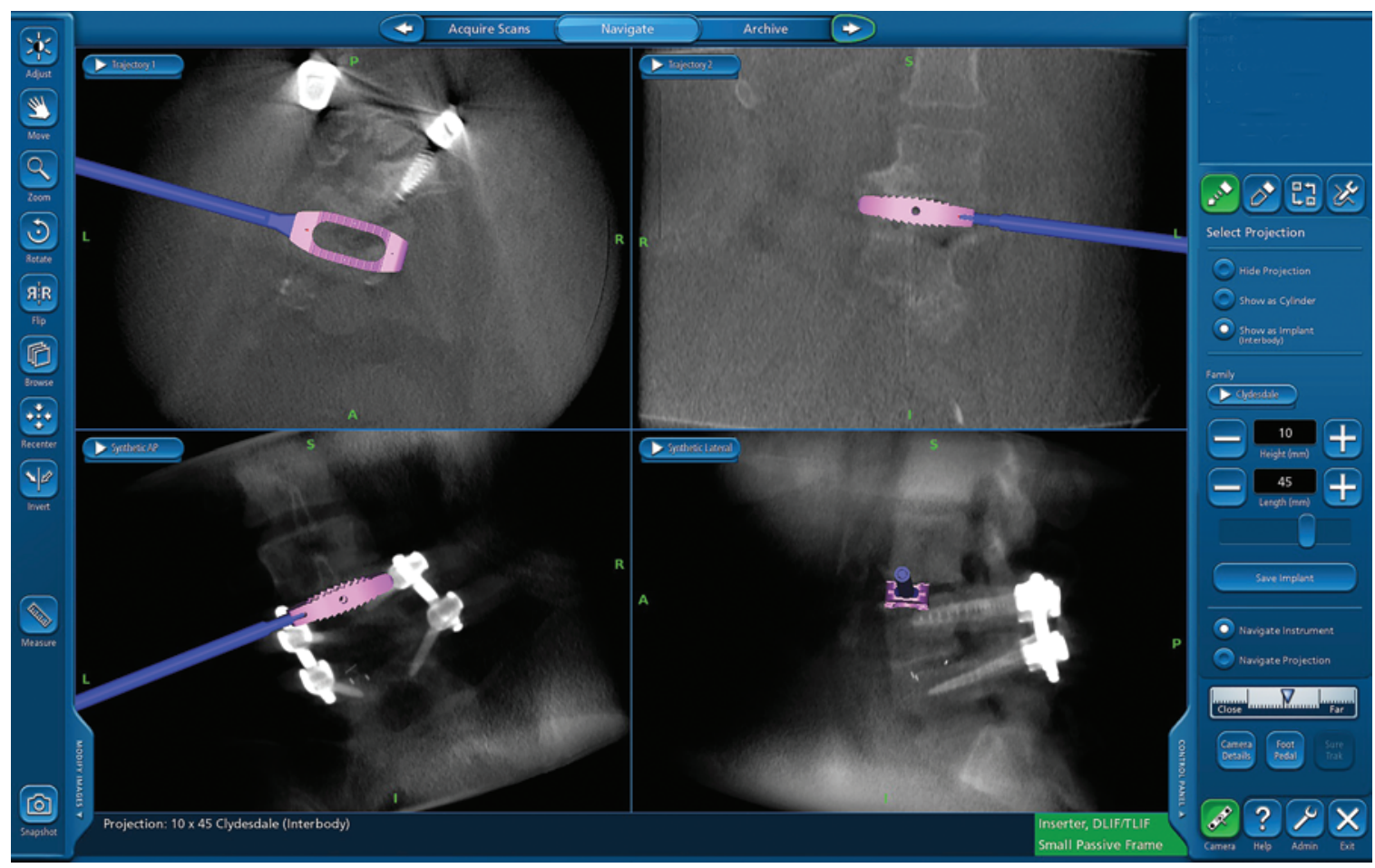

FIG. 4. Cage placement using IGNS. Cage position accuracy is verified by fluoroscopy. Figure is available in color online only. 

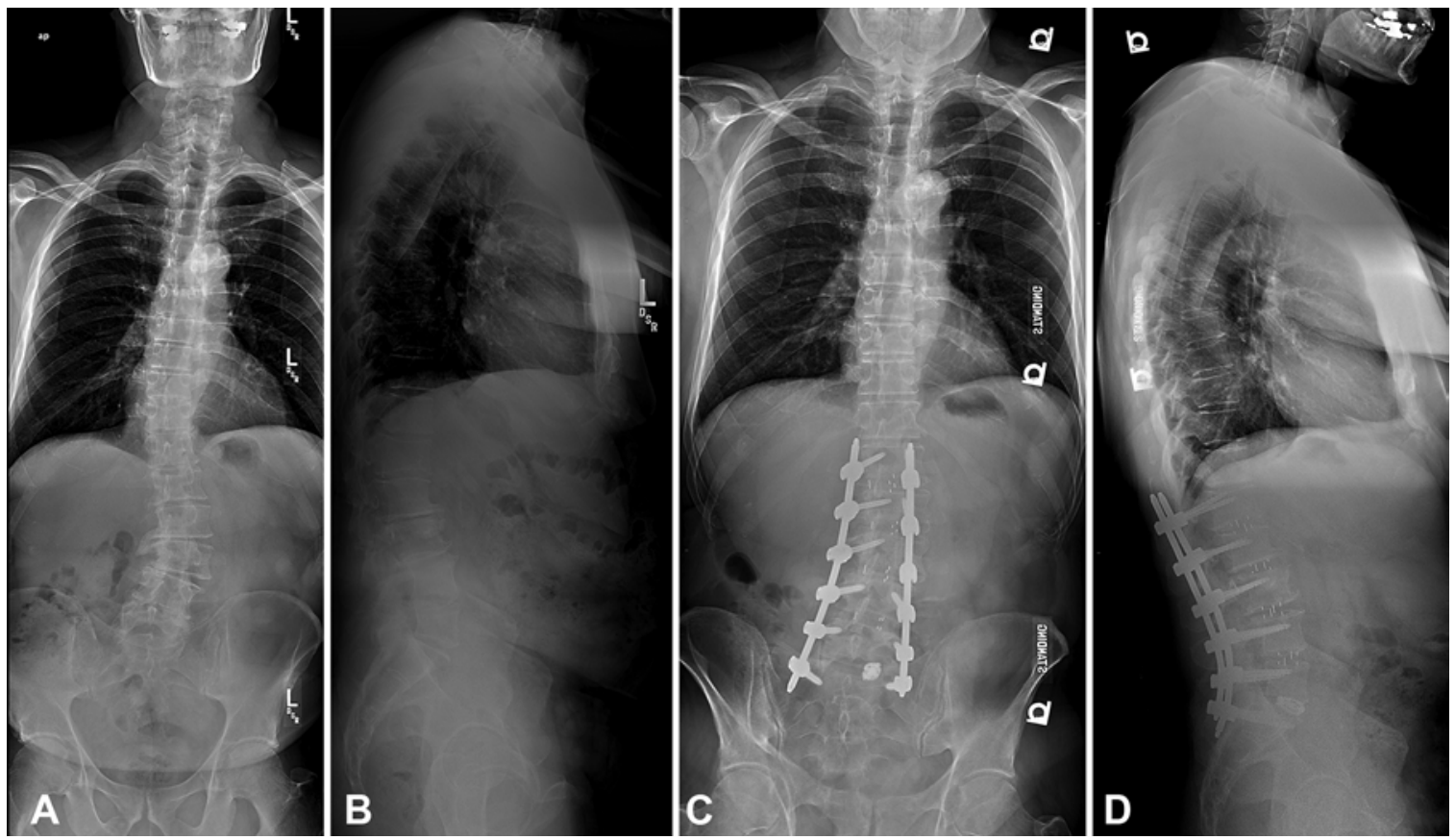

FIG. 5. Preoperative ( $A$ and $B$ ) and postoperative ( $C$ and $D)$ scoliosis radiographs obtained in a patient who underwent navigated L1-5 LLIF.

One patient developed a psoas muscle abscess. There were no further surgical-site infections. Three patients had mild (Grade $4+/ 5$ on the Medical Research Council criteria) residual hip flexion weakness at 1-month follow-up. Four patients had medical complications, including 2 patients with myocardial infarction, 1 of which was a non-ST-segment elevation associated with atrial fibrillation and rapid ventricular response. Both of these patients had a second-stage posterior procedure. The other 2 medical complications consisted of 1 patient who developed the Miller-Fisher variant of acute, intermittent demyelinating polyneuropathy, and another patient who developed urinary retention. All of these patients made a complete recovery with no residual effects. There was 1 patient in whom the attempted cage placement was aborted due to an endplate fracture that occurred during disc preparation.

The IGNS was used for each level fused. There were no instances of inaccuracy at any level in either the singlelevel or multilevel procedures. Postoperative imaging confirmed the expected placement of the cages based on the IGNS plan. Twenty-eight cages $(42.4 \%)$ were placed that spanned Quarters 1 to 2, 36 cages (54.5\%) spanned Quarters 2 to 3 , and 2 cages (3.0\%) spanned Quarters 3 to 4.

\section{Discussion}

Fluoroscopy is traditionally used for minimally invasive spinal procedures to guide the placement of retractors, as well as implants including screws and/or cages. However, the use of iCBCT and IGNS has recently become favored for a variety of techniques. It has been well studied for posterior approaches, with noted benefits in decreasing both operator and staff radiation exposure, as well as increasing accuracy.,4,10,15 The technical details and nuances of using an IGNS for LLIF have previously been reported..$^{3,12}$ Here, we present the results of a cohort of 31 consecutive patients who underwent LLIF using iCBCT and IGNS. There were no instances of significant inaccuracy or misguidance using this technique for either single-level or multilevel procedures.

For multilevel procedures, we elected to proceed from proximal to distal segments. This was done to minimize inaccuracy from cage placement with the assumption that the disc levels closer to the iliac reference pin would be less affected. No repeat iCBCTs were performed for multilevel cases. We did not encounter any significant problems with accuracy during multilevel LLIF utilizing the proximalto-distal sequence of cage placement. Of note, submillimeter accuracy was not achieved; in fact, at times there were errors that were approximately 1 to $3 \mathrm{~mm}$, which occurred during multilevel cases. However, this degree of accuracy was more than adequate for retractor positioning and accurate cage placement within the desired region of the disc space.

Previous anatomical studies have demonstrated that the lumbar plexus courses through the posterior quadrant of the disc space, though the genitofemoral nerve courses more anteriorly. ${ }^{2,13}$ Uribe et al. have suggested that disc entry in the middle or middle-posterior portion of the disc space represents a safe working zone during LLIF. ${ }^{13}$ In the present study, iCBCT and IGNS in conjunction with neurophysiological monitoring were used to place the retractors. There was no evidence of direct nerve injury, which suggests that this technique can be safely employed to avoid damage to the lumbosacral plexus.

Complication rates were acceptable and in line with previous reports. ${ }^{8}$ There were 9 approach-related complications noted in our cohort. These predominantly consisted of mild, transient paresthesia and hip flexion weakness. 
TABLE 1. Demographics and procedural data for the patient cohort $(n=31)$

\begin{tabular}{|c|c|c|c|c|c|c|c|c|c|}
\hline \multirow{2}{*}{$\begin{array}{l}\text { Case } \\
\text { No. }\end{array}$} & \multirow{2}{*}{$\begin{array}{l}\text { Age (yrs), } \\
\text { Sex }\end{array}$} & \multirow{2}{*}{$\begin{array}{c}\mathrm{BMl} \\
\left(\mathrm{kg} / \mathrm{m}^{2}\right)\end{array}$} & \multirow[b]{2}{*}{ Diagnosis } & \multirow[b]{2}{*}{ Side } & \multicolumn{4}{|c|}{ Cage Placement Quarters } & \multirow[b]{2}{*}{ Complications } \\
\hline & & & & & L1-2 & L2-3 & L3-4 & L4-5 & \\
\hline 1 & $57, \mathrm{M}$ & 35.0 & DS & $\mathrm{Lt}$ & $2-3$ & $2-3$ & NA & NA & NA \\
\hline 2 & $62, \mathrm{~F}$ & 40.2 & DS & Lt & NA & $1-2$ & $2-3$ & $1-2$ & NA \\
\hline 3 & $74, \mathrm{~F}$ & 22.3 & DS & $\mathrm{Lt}$ & $1-2$ & $2-3$ & $1-2$ & $2-3$ & NA \\
\hline 4 & $71, \mathrm{~F}$ & 25.7 & DS & Rt & $1-2$ & $1-2$ & $1-2$ & $1-2$ & Mild, residual thigh numbness \\
\hline 5 & $65, \mathrm{M}$ & 30.1 & DS & Rt & $2-3$ & $1-2$ & $2-3$ & $1-2$ & Acute, intermittent, Miller-Fisher demyelinating polyneuropathy \\
\hline 6 & $74, \mathrm{~F}$ & 20.9 & DS & Lt & NA & $1-2$ & $1-2$ & $2-3$ & Mild, residual hip flexion weakness \\
\hline 7 & $57, \mathrm{~F}$ & 28.3 & ASD & $\mathrm{Lt}$ & NA & NA & $2-3$ & NA & NA \\
\hline 8 & $73, \mathrm{~F}$ & 30.9 & ASD & Lt & NA & NA & $2-3$ & NA & NA \\
\hline 9 & $68, F$ & 47.1 & ASD & $\mathrm{Lt}$ & NA & NA & $1-2$ & NA & NA \\
\hline 10 & $51, F$ & 34.9 & PLKS & $\mathrm{Lt}$ & NA & $1-2$ & $1-2$ & $2-3$ & NA \\
\hline 11 & $69, \mathrm{~F}$ & 36.8 & DS & Rt & $3-4$ & $3-4$ & $2-3$ & $2-3$ & Transient hip flexion weakness \\
\hline 12 & $76, \mathrm{M}$ & 24.7 & DS & $\mathrm{Lt}$ & NA & NA & $2-3$ & NA & NA \\
\hline 13 & $64, \mathrm{~F}$ & 24.7 & ASD & $\mathrm{Lt}$ & $2-3$ & NA & NA & NA & Psoas abscess \\
\hline 14 & $69, F$ & 31.9 & DS & $\mathrm{Lt}$ & NA & NA & $1-2$ & NA & NA \\
\hline 15 & $54, \mathrm{~F}$ & 24.2 & DS & $\mathrm{Lt}$ & NA & $1-2$ & $2-3$ & $2-3$ & $\begin{array}{l}\text { Endplate fracture at L1-2 during disc preparation (level aborted) \& } \\
\text { transient hip flexion weakness }\end{array}$ \\
\hline 16 & $63, \mathrm{M}$ & 30.0 & PA & $\mathrm{Lt}$ & NA & $1-2$ & $1-2$ & NA & NA \\
\hline 17 & $66, \mathrm{~F}$ & 26.7 & DS & $\mathrm{Lt}$ & NA & $2-3$ & $2-3$ & $2-3$ & Mild, residual hip flexion weakness \\
\hline 18 & $63, \mathrm{M}$ & 30.1 & PLKS & $\mathrm{Lt}$ & NA & $2-3$ & $1-2$ & $1-2$ & Contralateral thigh numbness \\
\hline 19 & $73, \mathrm{M}$ & 37.4 & PA & $\mathrm{Lt}$ & NA & $2-3$ & $2-3$ & $1-2$ & Myocardial infarction \\
\hline 20 & $71, \mathrm{~F}$ & 25.6 & ASD & $\mathrm{Lt}$ & NA & NA & $2-3$ & NA & Mild residual thigh numbness \\
\hline 21 & $78, F$ & 24.2 & ASD & $\mathrm{Lt}$ & NA & NA & NA & $2-3$ & NA \\
\hline 22 & $73, \mathrm{~F}$ & 38.4 & DS & $\mathrm{Lt}$ & NA & $2-3$ & $2-3$ & NA & Mild, residual hip flexion weakness \\
\hline 23 & $55, \mathrm{~F}$ & 30.5 & ASD & $\mathrm{Lt}$ & NA & NA & $2-3$ & NA & NA \\
\hline 24 & $60, \mathrm{M}$ & 24.9 & PA & $\mathrm{Lt}$ & NA & $1-2$ & NA & NA & NA \\
\hline 25 & $61, F$ & 31.2 & ASD & $\mathrm{Lt}$ & NA & NA & NA & $2-3$ & NA \\
\hline 26 & $56, F$ & 38.3 & DS & $\mathrm{Lt}$ & NA & $1-2$ & $2-3$ & $2-3$ & NA \\
\hline 27 & $47, \mathrm{~F}$ & 33.5 & DDD & $\mathrm{Lt}$ & NA & NA & NA & $1-2$ & NA \\
\hline 28 & $79, \mathrm{M}$ & 26.4 & DS & Rt & NA & $2-3$ & $2-3$ & $2-3$ & Myocardial infarction \& atrial fibrillation \\
\hline 29 & $68, M$ & 34.9 & ASD & $\mathrm{Lt}$ & NA & $1-2$ & $1-2$ & NA & NA \\
\hline 30 & $24, \mathrm{M}$ & 35.2 & IS & $\mathrm{Lt}$ & NA & NA & $2-3$ & $2-3$ & Transient urinary retention \\
\hline 31 & $60, \mathrm{~F}$ & 37.1 & DDD & Rt & NA & NA & NA & $1-2$ & NA \\
\hline
\end{tabular}

$\mathrm{ASD}$ = adjacent-segment disease; $\mathrm{BMI}=$ body mass index; $\mathrm{DDD}$ = degenerative disc disease; $\mathrm{DS}$ = degenerative scoliosis; $I S$ = idiopathic scoliosis; NA = not applicable; PA = pseudarthrosis; PLKS = postlaminectomy kyphoscoliosis.

Of note, the lateral femoral cutaneous nerve (LFCN) traverses near the ASIS. While injury of the nerve is possible during placement, our iliac pin placement site is not near the typical course of the LFCN and so we feel the risk is minimal. It did not appear that there was significant injury to the LFCN in this cohort. Alternatively, the pin can be placed in the PSIS.

The issue of patient radiation exposure involved with iCBCT has been well studied.,16 Overall, iCBCT does not expose the patient to an excessive amount of radiation. As previously mentioned, each patient only had a single iCBCT used for navigation, and multiple images were not necessary.

This study has several limitations. The analysis was done retrospectively, which could induce biases, although all patients treated with image-guided spinal navigation were included to limit selection bias. In addition, accuracy was not graded independently, but rather was performed by the primary surgeons intraoperatively and subsequently further evaluated using postprocedure imaging.

\section{Conclusions}

LLIF can be safely and accurately performed utilizing iCBCT and IGNS. Accuracy is acceptable for multilevel procedures.

\section{References}

1. Abdullah KG, Bishop FS, Lubelski D, Steinmetz MP, Benzel EC, Mroz TE: Radiation exposure to the spine surgeon in lumbar and thoracolumbar fusions with the use of an intraoperative computed tomographic 3-dimensional imaging system. Spine (Phila Pa 1976) 37:E1074-E1078, 2012

2. Benglis DM, Vanni S, Levi AD: An anatomical study of 
the lumbosacral plexus as related to the minimally invasive transpsoas approach to the lumbar spine. J Neurosurg Spine 10:139-144, 2009

3. Drazin D, Liu JC, Acosta FL Jr: CT navigated lateral interbody fusion. J Clin Neurosci 20:1438-1441, 2013

4. Gelalis ID, Paschos NK, Pakos EE, Politis AN, Arnaoutoglou CM, Karageorgos AC, et al: Accuracy of pedicle screw placement: a systematic review of prospective in vivo studies comparing free hand, fluoroscopy guidance and navigation techniques. Eur Spine J 21:247-255, 2012

5. Graham RB, Wong AP, Liu JC: Minimally invasive lateral transpsoas approach to the lumbar spine: pitfalls and complication avoidance. Neurosurg Clin N Am 25:219-231, 2014

6. Knight RQ, Schwaegler P, Hanscom D, Roh J: Direct lateral lumbar interbody fusion for degenerative conditions: early complication profile. J Spinal Disord Tech 22:34-37, 2009

7. Lange J, Karellas A, Street J, Eck JC, Lapinsky A, Connolly PJ, et al: Estimating the effective radiation dose imparted to patients by intraoperative cone-beam computed tomography in thoracolumbar spinal surgery. Spine (Phila Pa 1976) 38:E306-E312, 2013

8. Lehmen JA, Gerber EJ: MIS lateral spine surgery: a systematic literature review of complications, outcomes, and economics. Eur Spine J 24 (Suppl 3):287-313, 2015

9. Nottmeier EW, Bowman C, Nelson KL: Surgeon radiation exposure in cone beam computed tomography-based, imageguided spinal surgery. Int J Med Robot 8:196-200, 2012

10. Nottmeier EW, Pirris SM, Edwards S, Kimes S, Bowman C, Nelson KL: Operating room radiation exposure in cone beam computed tomography-based, image-guided spinal surgery: clinical article. J Neurosurg Spine 19:226-231, 2013

11. Ozgur BM, Aryan HE, Pimenta L, Taylor WR: Extreme Lateral Interbody Fusion (XLIF): a novel surgical technique for anterior lumbar interbody fusion. Spine J 6:435-443, 2006

12. Park P: Three-dimensional computed tomography-based spinal navigation in minimally invasive lateral lumbar interbody fusion: feasibility, technique, and initial results. Neurosurgery 11 (Suppl 2):259-267, 2015

13. Uribe JS, Arredondo N, Dakwar E, Vale FL: Defining the safe working zones using the minimally invasive lateral retroperitoneal transpsoas approach: an anatomical study. J Neurosurg Spine 13:260-266, 2010

14. Uribe JS, Vale FL, Dakwar E: Electromyographic monitoring and its anatomical implications in minimally invasive spine surgery. Spine (Phila Pa 1976) 35 (26 Suppl):S368-S374, 2010

15. Van de Kelft E, Costa F, Van der Planken D, Schils F: A prospective multicenter registry on the accuracy of pedicle screw placement in the thoracic, lumbar, and sacral levels with the use of the O-arm imaging system and StealthStation Navigation. Spine (Phila Pa 1976) 37:E1580-E1587, 2012

16. Zhang J, Weir V, Fajardo L, Lin J, Hsiung H, Ritenour ER: Dosimetric characterization of a cone-beam O-arm imaging system. J XRay Sci Technol 17:305-317, 2009

\section{Disclosures}

The authors report the following: Dr. Park is a consultant for Medtronic, Globus Medical, and Biomet; receives royalties from Globus Medical; and receives non-study-related clinical/research support from Orthofix and the Blue Cross Blue Shield of Michigan Foundation. Dr. Patel is a consultant for Globus Medical and Stryker, as well as a paid speaker for Stryker.

\section{Author Contributions}

Conception and design: Park. Acquisition of data: all authors. Analysis and interpretation of data: all authors. Drafting the article: Joseph, Smith. Critically revising the article: Park. Reviewed submitted version of manuscript: all authors. Approved the final version of the manuscript on behalf of all authors: Park. Study supervision: Park.

\section{Correspondence}

Paul Park, Department of Neurosurgery, University of Michigan, 1500 East Medical Center Dr., Rm. 3552 TC, Ann Arbor, MI 48109-5338. email: ppark@med.umich.edu. 